\title{
Chaos in a Jahn-Teller Molecule
}

\author{
R.S. Markiewicz \\ Physics Department and Barnett Institute, Northeastern U., Boston MA 02115
}

The Jahn-Teller system $E \otimes b_{1} \oplus b_{2}$ has a particular degeneracy, where the vibronic potential has an elliptical minimum. In the general case where the ellipse does not reduce to a circle, the classical motion in the potential is chaotic, tending to trapping near one of the extrema of the ellipse. In the quantum problem, the motion consists of correlated tunneling from one extremum to the opposite, leading to an average angular momentum reminiscent of that of the better known $E \otimes e$ dynamic Jahn-Teller system.

In the well-known $E \otimes e$ Jahn-Teller (JT) effect, a molecule has a two-fold electronic degeneracy coupled to a doubly degenerate vibrational mode. This leads to a 'conical intersection' in the vibronic potential which has a degenerate, circular minimum ('Mexican hat potential'), although higher-order vibronic coupling can break the ring up into three degenerate minima along the trough ('tricorn potential') [1]. Quantum mechanically, the coupled electron-molecular vibrational (vibronic) wave function can tunnel between the three minima, leading to a ground state with a net angular momentum [2]. Remarkably, this 'orbital' angular momentum is quantized in half-integer multiples of $\hbar$, indicating the strong coupling of electronic and molecular motions. This quantization is a signature of the Berry phase [3,4] of $\pi$ associated with the dynamic Jahn-Teller effect; the $\pi$ Berry phase has been experimentally verified in triangular $\mathrm{Na}_{3}$ molecules [5]. Points of conical intersection lead to chaotic behavior in the vibrational spectra, manifested quantum mechanically by anomalous level statistics [6]. However, the high symmetry of the $E \otimes e$ problem precludes chaos [7], so multimode interactions must be included, and the chaos generally appears at high energies (above the conical intersection) where many vibrational modes are excited.

Here, it is shown that a simple modification of the symmetry preserves the anomalous Berry phase, yet leads to chaotic behavior at much lower energies, without the need of additional mode coupling. This case is the square $\mathrm{X}_{4}$ molecule with square planar symmetry, $D_{4 h}$, corresponding to an $E \otimes b_{1} \oplus b_{2}$ Jahn-Teller problem [1], Fig. 1. The high symmetry allows two JT modes, with independent frequencies $\omega_{i}, i=1,2$, and electron-vibration couplings $V_{i}$. In the special case $\omega_{1}=\omega_{2}, V_{1}=V_{2}$, the problem reduces exactly to that of the $E \otimes e$ molecule. However, there is an intermediate case, which seems not to have been explored till now. When $V_{1} / \omega_{1}=V_{2} / \omega_{2}$, the two modes have the same JT stabilization energy,
$E_{J T}^{(i)}=V_{i}^{2} / 2 M \omega_{i}^{2}$, and hence the vibronic potential has an elliptic minimum, which is not circular unless $\omega_{1}=\omega_{2}$. Given the elliptic minimum, the possibility of a periodic orbit arises. However, angular momentum is not conserved. In the present paper I analyze the resulting motion.

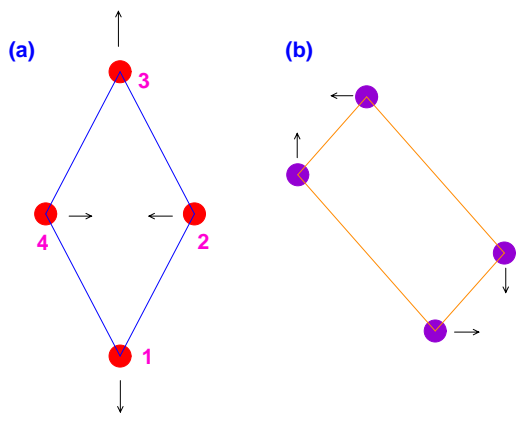

FIG. 1. $B_{1}$ (a) and $B_{2}$ (b) distortions of a square $\mathrm{X}_{4}$ molecule.

The phonon modes $B_{i}$ of amplitude $Q_{i}$ are defined as follows. The four atomic positions, Fig. 1 1 a, can be written as

$$
\begin{aligned}
& \vec{r}_{1}=\vec{r}_{10}-Q_{1} \hat{y}+Q_{2} \hat{x}, \\
& \vec{r}_{2}=\vec{r}_{20}-Q_{1} \hat{x}-Q_{2} \hat{y}, \\
& \vec{r}_{3}=\vec{r}_{30}+Q_{1} \hat{y}-Q_{2} \hat{x}, \\
& \vec{r}_{4}=\vec{r}_{40}+Q_{1} \hat{x}+Q_{2} \hat{y},
\end{aligned}
$$

where the $\vec{r}_{i 0}$ 's are the positions of the $\mathrm{X}$ atoms in the undistorted square. The vibronic interaction Hamiltonian is

$$
\begin{array}{r}
H_{v i b}=V_{1} Q_{1} T_{x}+V_{2} Q_{2} T_{y} \\
=V_{1} Q_{1}\left(\begin{array}{cc}
1 & 0 \\
0 & -1
\end{array}\right)+V_{2} Q_{2}\left(\begin{array}{ll}
0 & 1 \\
1 & 0
\end{array}\right) .
\end{array}
$$

Here the electronic operators are represented by the pseudospin $T_{i}$ 's and other factors are included in the electronphonon coupling $V_{i}$. To the vibronic Hamiltonian must be added an electronic term $H_{e l}$ and a phononic part $H_{p h}$, with

$$
H_{p h}=\frac{1}{2 M}\left(P_{1}^{2}+P_{2}^{2}+M^{2} \omega_{1}^{2} Q_{1}^{2}+M^{2} \omega_{2}^{2} Q_{2}^{2}\right)
$$

with $\omega_{i}$ the bare phonon frequencies. A spin-orbit coupling can be included [8]

$$
H_{s o}=\lambda \vec{L} \cdot \vec{S} .
$$


For a static JT effect, the momenta $P_{i}$ can be neglected, and the $Q_{i}$ are chosen to minimize the energy, Eqs. 2, 3. The solution can be written in terms of the JT energy $E_{J T}^{(i)}=V_{i}^{2} /\left(2 M \omega_{i}^{2}\right)$. For $E_{J T}^{(1)} \neq E_{J T}^{(2)}$, the lowest energy state consists of a distortion of the mode with larger JT energy only. For instance, if $\left.E_{J T}^{(2)}>E_{J T}^{(1}\right)$, the solution is $Q_{1}=0, Q_{2}=V_{2} /\left(M \omega_{2}^{2}\right), E=-E_{J T}^{(2)}$.

Special cases arise when

$$
E_{J T}^{(1)}=E_{J T}^{(2)} \equiv E_{J T} .
$$

Eliminating the electrons produces the vibronic potential surfaces

$$
E_{ \pm}=\frac{M}{2}\left(\omega_{1}^{2} Q_{1}^{2}+\omega_{2}^{2} Q_{2}^{2}\right) \pm \sqrt{V_{1}^{2} Q_{1}^{2}+V_{2}^{2} Q_{2}^{2}+\frac{\lambda^{2}}{4}} .
$$

When Eq. 5 is satisfied, the lower vibronic surface has a minimum which is degenerate along a trough, similar to the Mexican hat:

$$
\begin{aligned}
& Q_{1}^{0}=Q_{0}^{0} \frac{\cos \theta}{\omega_{1}} \\
& Q_{2}^{0}=Q_{0}^{0} \frac{\sin \theta}{\omega_{2}}
\end{aligned}
$$

with $Q_{0}^{0}=\sqrt{2 E_{J T}-\lambda^{2} / 8 E_{J T}}$, and $\theta$ arbitrary. Near the trough, the lower potential surface can be expanded:

$$
E_{-}=\frac{M}{2} \alpha(\vec{g} \cdot \vec{q})^{2}
$$

with $\vec{q}=\left(q_{1}, q_{2}\right), q_{i}=Q_{i}-Q_{i}^{0}, \alpha=1-\lambda^{2} / 16 E_{J T}^{2}$ and $\vec{g}=\left(\omega_{1} \cos \theta, \omega_{2} \sin \theta\right)$ - that is, there is a restoring force only 'perpendicular' to the trough. Defining $\beta_{\omega}=\omega_{2} / \omega_{1}$, the electronic eigenvectors are

$$
\begin{array}{r}
\psi_{+}=\cos \gamma \psi_{1}+\sin \gamma \psi_{2} \\
\psi_{-}=-\sin \gamma \psi_{1}+\cos \gamma \psi_{2},
\end{array}
$$

where $\tan \gamma=\left(\sqrt{1+\delta \sin ^{2} \theta}-\cos \theta\right) /\left(\beta_{\omega} \sin \theta\right)$ and $\delta=$ $\beta_{\omega}^{2}-1$, Fig.2. By convention, $\omega_{2}$ is assumed to be the higher frequency $\left(\beta_{\omega} \geq 1\right)$.

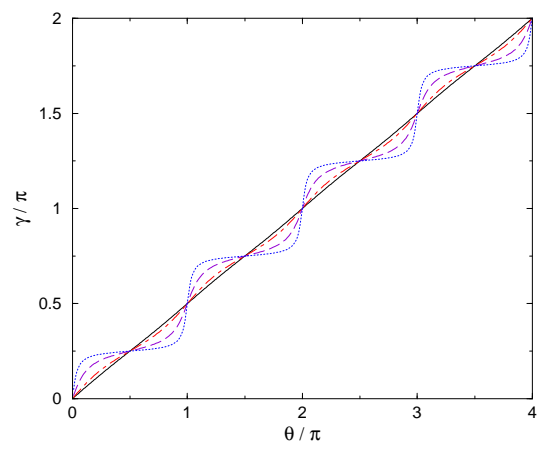

FIG. 2. Electronic phase $\gamma$ vs phononic phase $\theta$, for $\delta=$ 0.1 (solid line), 1 (dotdashed line), 10 (dashed line), and 100 (dotted line).
If additionally $\omega_{1}=\omega_{2}$, the problem reduces to the $E \otimes e$ problem, and in Eq. $9 \gamma=\theta / 2$ : the electronic wave function is double valued: when $\theta$ changes by $2 \pi, \gamma$ has only changed by $\pi$ (the wave functions have changed sign). This sign change is the signature of a Berry phase [3, 4 , and causes the vibronic orbital angular momentum to take on half-integer values [2]. This can be seen as follows [1]. The z-component of orbital angular momentum is $L_{z}=\left(Q_{1} P_{2}-Q_{2} P_{1}\right) / \hbar$, and the operator which commutes with the vibronic hamiltonian, Eq. 2 is

$$
j_{z}=L_{z}+\frac{1}{2} T_{z}
$$

Since $L_{z}$ is quantized in integers, $j_{2}$ has half-integral quanta. Note from Fig. 2 that even when $\omega_{1} \neq \omega_{2}, \theta$ must change by $4 \pi$ to produce a $2 \pi$ change in $\gamma$, suggesting a similar Berry phase. This can be directly demonstrated. The Berry phase is [9]

$$
\begin{array}{r}
\gamma_{B}=-s \int_{0}^{2 \pi} \frac{\partial \gamma}{\partial \theta} d \theta \\
=-\beta s \int_{0}^{2 \pi} \frac{d \theta}{1+\sin ^{2} \theta}=-2 \pi s,
\end{array}
$$

where $s$ is half an odd integer, introduced to make the total wave function single valued. Thus the Berry phase is $\pi$, modulo $2 \pi$, for any anisotropy.

While this is a standard JT problem, I have not found any detailed analysis of the limit Eq. 5. As a first step, I perform a canonical transformation

$$
H^{\prime}=e^{i S} H e^{-i S}=H+i[S, H]-\ldots
$$

with

$$
S=-\left(\frac{V_{1}}{\omega_{1}^{2}} P_{1} T_{x}+\frac{V_{2}}{\omega_{2}^{2}} P_{2} T_{y}\right) .
$$

The canonical transformation can be performed exactly [10], but for present purposes only the first order result is needed. S, Eq. 13, was chosen to exactly cancel the term linear in $Q$. It yields a correction

$$
H_{2}^{\prime}=i\left[S, H_{v i b}\right]=-\frac{T_{0}}{2}\left(\frac{V_{1}^{2}}{\omega_{1}^{2}}+\frac{V_{2}^{2}}{\omega_{2}^{2}}\right)+\frac{V_{1} V_{2}}{\omega_{1}^{2} \omega_{2}^{2}} A T_{z},
$$

with $T_{0}$ the identity matrix,

$$
A=\omega_{2}^{2} P_{1} Q_{2}-\omega_{1}^{2} P_{2} Q_{1}=-\omega_{+}^{2} L_{z}+\omega_{-}^{2}\left(P_{1} Q_{2}+P_{2} Q_{1}\right),
$$

and $\omega_{ \pm}^{2}=\left(\omega_{1}^{2} \pm \omega_{2}^{2}\right) / 2$. Thus, when $\omega_{-}=0, H_{2}^{\prime}$ is proportional to $L_{z} T_{z}$, and the angular momentum $j_{z}=$ $L_{z}+T_{z} / 2$ is conserved (Eq. 10). For the present case $\omega_{-} \neq 0$ and $j_{z}$ is not constant.

Given the presence of a circular trough in the potential, circulating orbits should be possible: could it be that 
there is a nonvanishing average $\left\langle j_{z}>\neq 0\right.$ even though $j_{z}$ is not constant? This possibility can be explored in the related classical Hamiltonian (particle in a non-linear potential well) by numerically integrating the equations of motion

$$
\begin{array}{r}
\ddot{Q}_{i}=-\frac{d E_{-}}{d Q_{i}}=-\omega_{i}^{2} Q_{i}\left(1-\frac{2 E_{J T}}{\sqrt{V_{1}^{2} Q_{1}^{2}+V_{2}^{2} Q_{2}^{2}+\lambda^{2} / 4}}\right) \\
\simeq-\alpha \omega_{i}^{2} Q_{i}\left(1-\frac{q_{0}}{\sqrt{\omega_{1}^{2} Q_{1}^{2}+\omega_{2}^{2} Q_{2}^{2}}}\right)
\end{array}
$$

where the last form utilizes the quadratic approximation, Eq. 8, dots indicate time derivatives, and $q_{0}^{2}=$ $2 E_{J T} \alpha$. The integral is evaluated using a Runge-Kutta routine with initial conditions $\vec{Q}(0)=\left(q_{0} / \omega_{1}, 0\right), \dot{\vec{Q}}(0)=$ $\left(0, \beta q_{0} / \omega_{2}\right)$. In the remaining analysis, I take $\lambda=0$.

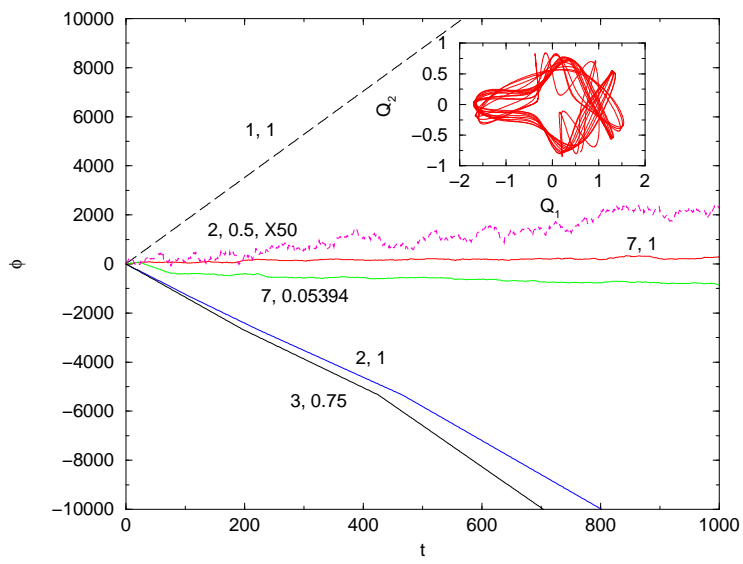

FIG. 3. Winding angle $\phi$ calculated from Eq. 17, for several values of $\left(\beta_{\omega}, \beta\right)$. Inset $=$ time series, $Q_{1}, Q_{2}(t)$ for $\beta_{\omega}=\omega_{2} / \omega_{1}=2, \beta=0.5$.

Given $Q_{1}(t), Q_{2}(t)$, a winding angle $\phi$ is defined such that

$$
\dot{\phi}=\frac{Q_{1} \dot{Q}_{2}-Q_{2} \dot{Q}_{1}}{Q_{1}^{2}+Q_{2}^{2}} .
$$

If one applies this procedure to the $E \otimes e$ problem $\left(\omega_{2}=\omega_{1}\right)$, the results are quite simple (long dashed line in Fig. 3): $\phi$ increases linearly with time, although the frequency is not constant, but varies approximately logarithmically with the velocity parameter $\beta$. By contrast, when $\omega_{2} \neq \omega_{1}$ Figure 3 shows that $\phi$ is generically a random function of time, with no linearly increasing part indicative of a non-zero $\left\langle j_{z}\right\rangle$. The various data sets are characterized by the two parameters $\left(\omega_{2} / \omega_{1}, \beta\right)$. (The figure utilizes the exact form of Eq. 16: the approximate form yields equivalent results.) The figure also clearly suggests that the motion is chaotic. This is further indicated by the direct time series, inset of Fig. 3 .

On the other hand, there are certain special values of the initial conditions for which the motion is approximately periodic, and $\phi$ does increase linearly with time.
These values may most easily be found by plotting $\phi(T)$ vs $\beta$ for some long time $T$. Typical examples are illustrated in Fig. 3, while the time series are shown in Fig. 1. Poincare maps (plots of $Q_{1}$ vs $\dot{Q}_{1}$ when $Q_{2}=0$ ), Figure 5, confirm the chaotic nature. (Note that the curve $(7,0.05394)$ is almost periodic - see particularly $Q_{1}(t)$, Fig. $1 \mathrm{~d}$ - but the Poincare map is clearly chaotic, Fig. 5d.) While the $E \otimes e$ limit, $\beta_{\omega}=1$, is quasiperiodic (the Poincare map is a smooth closed curve), for $\beta_{\omega} \neq 1$ even the special values are weakly chaotic, with the Poincare maps, Fig. 5 a, having a finite spread away from smooth curves. The similarity of these special trajectories to scars in e.g., Sinai stadia [11] should be noted.
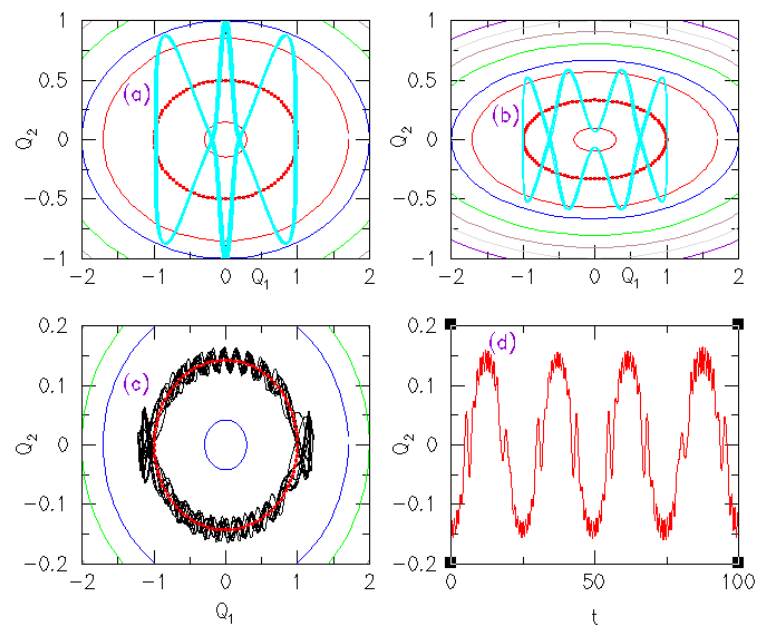

FIG. 4. Time series, $Q_{2}(t)$ vs $Q_{1}(t)$ (or vs t, in (d)) for several choices of $\left.\beta_{\omega}, \beta\right)$ : (a) $=(2,1),(b)=(3,0.75),(\mathrm{c}, \mathrm{d})$ $=(7,0.05394)$. In frames $(\mathrm{a}-\mathrm{c})$, the ellipses are equipotential contours, with the beaded contour representing the potential minimum.
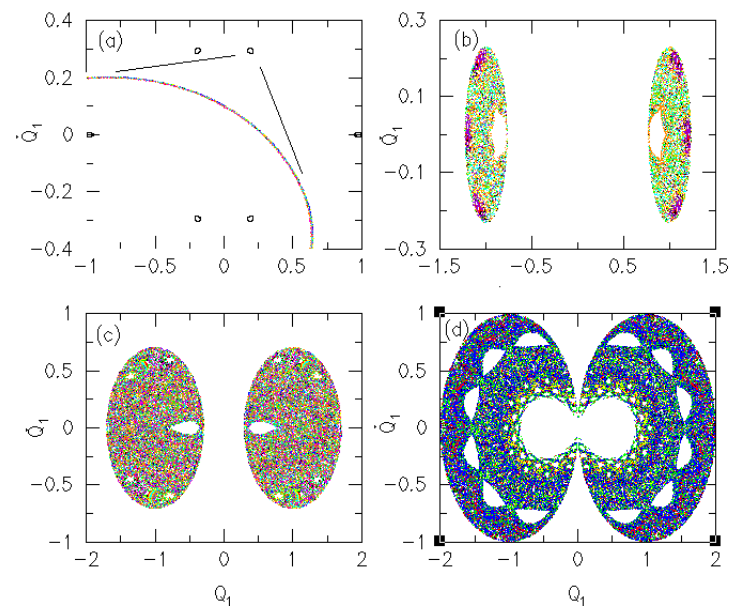
FIG. 5. Poincare maps for $\left(\beta_{\omega}, \beta\right)=(2,1)$ (a), $(7,0.05394)$ (b), $(2,0.5)(\mathrm{c}),(7,1)(\mathrm{d})$. In (a), one attractor is shown blown up.

How is this chaotic behavior manifested in the quantum limit? To explore this, it is convenient to first rescale the variables, so that the potential has circular symmetry, and the anisotropy appears in the ionic mass, $m_{i}=M\left(\omega_{0} / \omega_{i}\right)^{2}$, with $\omega_{0}^{2}=\left(\omega_{1}^{2}+\omega_{2}^{2}\right) / 2$, and then reduce the problem to one dimension by assuming that the motion is confined to the bottom of the trough and only $\phi$ varies. The Hamiltonian becomes $H=-\hbar^{2} h /\left(2 m_{+} \rho_{0}^{2}\right)$, where $\rho_{0}$ is the equillibrium trough radius, $m_{ \pm}^{-1}=\left(m_{2}^{-1} \pm m_{1}^{-1}\right) / 2$ and

$$
h=\partial_{\phi}^{2}+\hat{\alpha}\left[\cos 2 \phi\left(\frac{3}{2}-\partial_{\phi}^{2}\right)+3 \sin 2 \phi \partial_{\phi}\right]-A_{4} \cos 4 \phi,
$$

with $\hat{\alpha}=m_{+} / m_{-}=\left(\beta_{\omega}^{2}-1\right) /\left(\beta_{\omega}^{2}+1\right)$ and higher order vibronic effects are incorporated in the term proportional to $A_{4}$. Schroedinger's equation can be integrated numerically, letting $\psi(\phi, t)=\psi(j \epsilon, n \delta) \equiv \psi_{j}^{n}$, with $\partial_{\phi} \psi=\left(\psi_{j+1}^{n}-\psi_{j}^{n}\right) / \epsilon$, and [12]

$$
\psi_{j}^{n+1}=e^{-i H \delta / \hbar} \psi_{j}^{n} \simeq \frac{1-i H \delta / 2 \hbar}{1+i H \delta / 2 \hbar} \psi_{j}^{n},
$$

or finally $(1-i \gamma h) \psi_{j}^{n+1}=(1+i \gamma h) \psi_{j}^{n}$, with $\gamma=$ $\hbar \delta / 4 m_{+} \rho_{0}^{2}$.

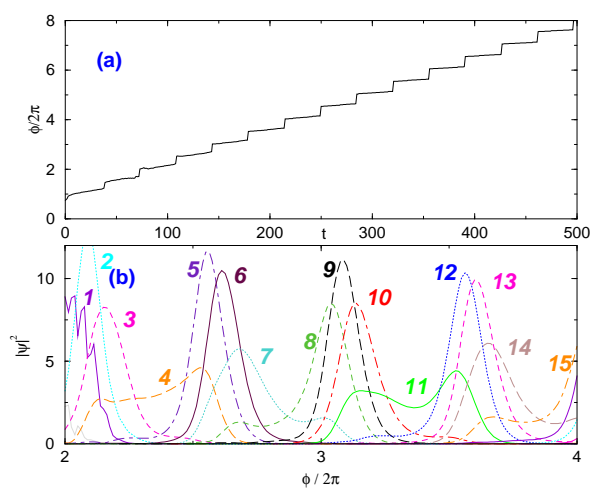

FIG. 6. Quantum time evolution, showing (a) position of wave function peak as a function of time, and (b) actual distribution of $|\psi|^{2}$ at several equally-spaced time intervals $\left(\hat{\alpha}=0.3, A_{4}=0\right)$.

Equation 19 was integrated numerically, assuming an initial gaussian distribution. Figure $6 \mathrm{~b}$ shows $|\psi(\phi, t)|^{2}$ for a variety of times $t$. The data can be better understood from Fig. 6a, which plots $\phi_{\max }$ vs $t$, where $\phi_{\max }$ is that value of $\phi$ for which $|\psi|^{2}$ has its maximum value. The wave function remains trapped in one of the effective potential wells, then quickly hops to the next one in a relatively short time. This hopping takes place by the probability spreading over two adjacent wells, as shown in Fig. 6 b at times 4, 7, and 11. The tunneling is coherent, so there is a net circulation. Additional information can be found by analyzing the Husimi density 13 $\rho_{H}(p, q)=|\langle p, q \mid \psi\rangle|^{2}$, with

$$
<p, q \mid \psi>=\sqrt[4]{\frac{s}{\hbar \pi}} \int \exp \left[-\frac{s(\phi-q)^{2}}{2 \hbar}-i \frac{p}{\hbar}\left(\phi-\frac{q}{2}\right)\right] \psi(\phi) d \phi,
$$

which describes the approximate smearing of $\psi$ in $q$ and $p$ as a function of time. Typical results are shown in Fig. 7, for squeezing parameter $s=1$.

Thus, the quantum system shows a 'memory' of the classical chaos, in that the wave function shows similar trapping near the points $Q_{2}=0$. However, whereas the wavefunctions appear to vary stochastically from cycle to cycle, Fig. 6b, the average of the wave function progresses smoothly, Fig. 6a. The main difference is that classically, the wave function can be reflected from a trapping region, reversing its direction of motion, while the quantum wave function always moves in the same direction, similar to the classical problem with special initial conditions. It seems plausible to interpret the special choice of initial conditions as analogous to a Bohr-Sommerfeld quantization condition in the quantum problem.

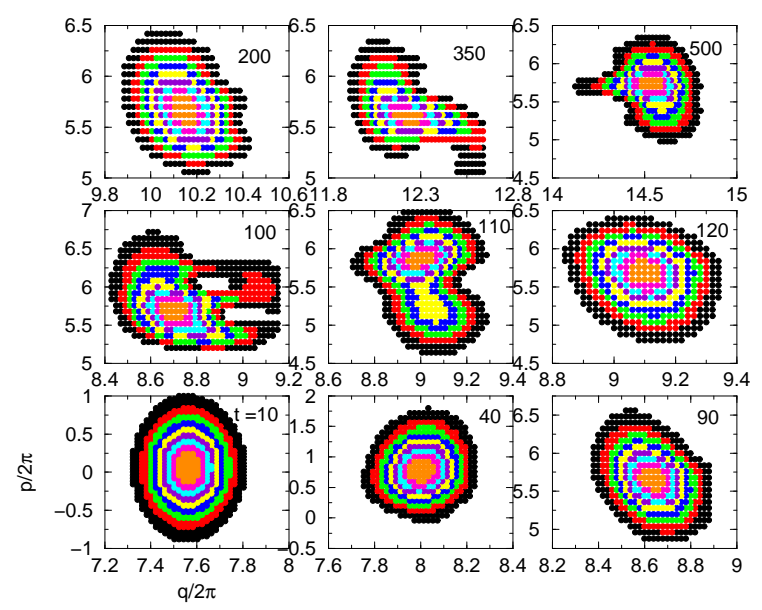

FIG. 7. Contour plot of Husimi distribution $\rho_{H}$ of data similar to that of Fig. 6 at several time intervals. An interwell hopping event occurs between times 90 and 120 .

As shown in Fig. 6a, the position of the wave function peak has a step-like component superposed on an average shift with time. This average shift is independent of the mass anisotropy 15, hence corresponding to the same quantized angular momentum as in the isotropic case. This is consistent with the Berry phase remaining $\pi$, Eq. 11, but somewhat surprising in light of the classical chaos. Blumel [16] has suggested that this might be a manifestation of quantum localization in angular momentum space [17, while the classical problem leads to 
angular momentum space diffusion. This possibility will be explored in future work.

In addition to its molecular interest, the present results may have condensed matter applications. Berryonic matter 114 has been postulated to explain anomalous properties of Buckyballs and other dynamic JT systems, but based on unit cells of triatomic molecules. Potential applications are greatly expanded for bases of square molecules [15].

I thank J. Jose, F.S. Ham, R. Englman, R. Blumel, and B. Barbiellini for stimulating conversations.

[1] I.B. Bersuker and V.Z. Polinger, "Vibronic Interactions in Molecules and Crystals" (Springer, Berlin, 1989); M.D. Kaplan and B.G. Vekhter, "Cooperative Phenomena in Jahn-Teller Crystals" (Plenum, N.Y., 1995).

[2] G. Herzberg and H.C. Longuet-Higgins, Disc. Farad. Soc. 35, 77 (1963).

[3] C.A. Mead, Rev. Mod. Phys. 64, 51 (1992).

[4] M.V. Berry, Proc. Roy. Soc. London, A392, 45 (1984).

[5] H. von Busch, Vas Dev, H.-A. Eckel, S. Kasahara, J. Wang, W. Demtröder, P. Sebald, and W. Meyer, Phys. Rev. Lett. 81, 4584 (1998).

[6] E. Haller, H. Köppel, and L.S. Cederbaum, J. Molec. Spectrosc. 111, 377 (1985); A. Delon, R. Jost, and M. Lombardi, J. Chem. Phys. 95, 5701 (1991).

[7] H. Köppel, W. Domcke, and L.S. Cederbaum, Advanc. Chem. Phys. 57, 59 (1984).

[8] C.J. Ballhausen, Theoret. Chim. Acta (Berl.) 3, 368 (1965).

[9] J.W. Zwanziger and E.R. Grant, J. Chem. Phys. 87, 2954 (1987).

[10] M. Wagner, in "The Dynamical Jahn-Teller Effect in Localized Systems", ed. by Yu.E. Perlin and M. Wagner (North Holland, Amsterdam, 1984), p. 155.

[11] S. Sridhar, Phys. Rev. Lett. 67, 785 (1991); A. Kudrolli, S. Sridhar, A. Pandey, and R. Ramaswamy, Phys. Rev. E49, 11 (1994).

[12] H.J. Korsch and H. Wiescher, in "Computational Physics", edited by K.H. Hoffman and M. Schreiber (Springer, Berlin, 1996), p. 225.

[13] K. Husimi, Proc. Phys. Math. Soc. Jpn. 22, 264 (1940).

[14] N. Manini, E. Tosatti, and S. Doniach, Phys. Rev. B51, 3731 (1995).

[15] R.S. Markiewicz and C. Kusko, unpublished.

[16] R. Blumel, personal communication.

[17] F. Borgonovi, G. Casati, and B. Li, Phys. Rev. Lett. 77, 4744 (1996); K.M. Frahm and D.L. Shepelyansky, Phys. Rev. Lett. 78, 1440 (1997). 